\title{
Etude des Equations des Fluides Chargés Relativistes Inductiifs et Conducteurs
}

\author{
YVonne BruHat \\ Institut H. Poincaré, 11 Rue P. Curie, Paris \\ Reçu le 28 Février 1966
}

\begin{abstract}
In the first part a system of equations for an inductive charged relativistic fluid with finite conductivity is written in a space time with given metric, taking into account thermodynamic phenomena. Speeds of propagation of various types of waves are determined under a restrictive hypothesis concerning the heat current $q$ : that $q$ depends only on the thermodynamical quantities and the gradient of one function of these quantities.

In the second part it is shown, by a detailed study of the characteristic polynomial and of its irreducible factors, that, when $q$ is negligible, the proposed system is non-strictly hyperbolic in the sense of J. LERAY and Y. OHYA and existence and uniqueness theorems of a certain Gevrey class are verified; the relativistic causality principle is satisfied under some physically reasonable assumptions on the thermodynamical quantities. The system becomes strictly hyperbolic (existence and uniqueness theorems obtain in classes of functions with a finite number of derivatives) when the fluid is both non inductive and of zero electrical conductivity.

In the third part we show briefly, by the methods of the second part, that the equations of relativistic fluids, with an infinite electrical conductivity is also nonstrictly hyperbolic. The linearized equations (in the neighborhood of constant values) are strictly hyperbolic.
\end{abstract}

\section{Introduction}

Il est difficile d'écrire les équations des fluides chargés inductifs en Relativité Générale: le tenseur de Maxwell pour de tels fluides, tel qu'il nous est fourni par la théorie classique, est en effet non symétrique et ne peut donc pas être ajouté tel quel au deuxième membre des équations d'Einstein. De nombreuses tentatives ont été faites pour symétriser ce tenseur (voir par exemple [1], [2], [3]) et interpréter le terme ainsi ajouté comme interaction entre l'impulsion énergie matérielle et le champ électromagnétique: l'addition de tels termes modifie évidemment les équations du mouvement ${ }^{1}$ et conduit, pour les divers essais qui ont été faits, à des résultats assez peu satisfaisants: d'une part les équations obtenues ont un aspect mathématique compliqué, le polynôme carac-

1 Contrairement à l'opinion parfois énoncée qu'il suffit, pour ne pas les modifier, de prendre le terme complémentaire à divergence nulle: une telle condition est ellemême, ici, une restriction sur les grandeurs à déterminer. 
téristique en un point semble irréductible et il est impossible de l'étudier autrement que par des approximations (cf. [2]), d'autre part, dans ces tentatives de symétrisation, soit l'énergie électromagnétique soit la force de Lorentz, ne coïncident pas dans un repère propre avec les valeurs admises par la physique classique (relativité restreinte). Les considérations qui précèdent ont conduit Latrémolière [4] et PichoN [5] à renoncer à la symétrisation du tenseur de Maxwell et au couplage avec les équations d'Einstein en prenant pour équations des fluides chargés inductifs, dans une métrique donnée, la généralisation directe des équations de ces fluides en relativité restreinte,

$$
\nabla_{\alpha} T^{\alpha \beta}=0 \text {. }
$$

La justification physique d'un tel procédé (cf. C. LATRÉmoLIغ̀nE [4]) est que le champ de gravitation, dans un domaine assez petit, ne dépend pratiquement pas de la matière dans ce domaine, mais de toute celle présente dans les régions éloignées (par exemple dans un laboratoire terrestre le champ de gravitation ne dépend pratiquement pas des phénomènes qui s'y déroulent); la justification mathématique en est dans la possibilité d'une étude mathématique complète des équations obtenues: G. Pichon a montré que, si l'on néglige les phénomènes thermodynamiques, le polynôme caractéristique se décompose en un produit de polynômes du $2^{e}$ degré au plus et il a déterminé explicitement les ondes et vitesses de propagation correspondantes, par l'étude du problème de Cauchy analytique.

Nous nous proposons dans la première partie de cet article de reprendre l'étude des équations des fluides chargés inductifs, dans une métrique donnée (qui peut être la métrique de Minkovski)

$$
\nabla_{\alpha} T^{\alpha \beta}=0
$$

en tenant compte des phénomènes thermodynamiques. Nous considérons d'abord le cas général où la conductibilité thermique n'est pas nulle: nous ajoutons alors au tenseur d'impulsion énergie un tenseur d'origine thermique non symétrisé ${ }^{2}$ : le principe de Taub de conservation de la matière impose alors une équation de continuité, qu'on peut interpréter comme équation de conservation de l'énergie (ou équation de la chaleur). Nous déterminons explicitement les diverses ondes et vitesses de propagation correspondantes sous une hypothèse très large faite sur le courant de chaleur $q$, à laquelle nous sommes conduits par l'étude même des équations, à savoir qu'il ne dépend que des "grandeurs d'état« et du gradient d'une fonction de ces grandeurs (que l'on pourrait peut-être interpréter comme la température absolue).

${ }^{2}$ Cf. à ce sujet Pham Man Quan [6] ainsi que Y. Bruhat et Pham MaN QUAN [7]. 
Dans une deuxième partie, nous montrons, à l'aide d'une étude détaillée du déterminant caractéristique et de ses facteurs irréductibles, que, dans le cas où la conductibilité thermique est négligeable les équations que nous avons obtenues pour les fluides chargés inductifs forment un système hyperbolique non strict au sens de Leray et OHYA [8], [9]: des théorèmes d'existence et d'unicité sont valables pour de tels systèmes dans des classes de Gevery (fonctions indéfiniment dérivables dont les dérivées satisfont à certaines majorations, de telles fonctions peuvent être à support compact). Dans le cas étudié l'indice $\alpha$ de Gevrey ( $\alpha$ caractérise la classe de Gevrey, qui est analytique pour $\alpha=1$, qui est une classe de Sobolev, fonctions à un nombre fini de dérivées, pour $\alpha=\infty)$ trouvé est $\alpha=2$. Les solutions ont un domaine d'influence déterminé par le cône fondamental de la métrique sous certaines inégalités, physiquement raisonnables, vérifiées par les grandeurs d'état.

Toute l'étude faite dans ces deux parties concerne les fluides de conductivité électrique finie. Les résultats concernant le théorème d'existence ne sont pas améliorés si les fluides ne sont pas inductifs $(\varepsilon \mu=1)$ ou si la conductivité s'annulle $(\sigma=0)$ : par contre si l'on a simultanement $\varepsilon \mu=1$ et $\sigma=0$ le système devient hyperbolique strict $(\alpha=\infty)$ : on retrouve ainsi un résultat de travaux antérieurs [10], [11] mais sans supposer la constance dans le fluide du rapport $\gamma / r$.

Dans une brève troisième partie nous montrons comment l'application des méthodes de la $2^{\text {ème }}$ partie permet de montrer le caractère hyperbolique non strict $(\alpha=3 / 2)$ des équations des fluides relativistes de conductivité électrique infinie (cf. [3], [14]) et conductibilité thermique nulle, conformément au résultat obtenu par LichNerowicz [14] par une diagonalisation directe.

\section{Equations des fluides chargés inductifs et conducteurs. Ondes et vitesses} de propagation

\section{Equations}

Nous écrirons ces équations sur une variété différentiable $V_{4}\left(C^{\infty}\right)$, munie d'une métrique riemanienne hyperbolique normale, à 1 carré $>0$ et 3 carrés $<0$

$$
d s^{2}=g_{\alpha \beta} d x^{\alpha} d x^{\beta}, \quad \alpha, \beta=0,1,2,3
$$

(qui peut être la métrique de Minkovski en coordonnées quelconques).

Nous prendrons pour tenseur d'impulsion énergie d'un fluide chargé inductif le tenseur non symétrisé

$$
T_{\alpha \beta}=r f u_{\alpha} u_{\beta}-p g_{\alpha \beta}+\tau_{\alpha \beta}-q_{\alpha} u_{\beta}
$$

où, selon le point de vue de TaUb [12], repris par Pichon [5] et LichNEROWICZ [11], $r$ est la densité matérielle propre, $f$ l'indice du fluide

$$
f=1+\mathscr{E}+\frac{p}{r},
$$


où $i=\mathscr{E}+\frac{p}{r}$ est l'enthalpie spécifique, $\mathscr{E}$ l'énergie interne spécifique, $p$ est la pression, $u_{\alpha}$ le vecteur vitesse unitaire (dans la métrique (1.1))

$$
u^{\alpha} u_{\alpha}=1 \text {. }
$$

Les quantités $r, f, p$ sont les "grandeurs d'état《 que nous supposerons connues en fonction de deux d'entre elles, par exemple $f$ et $p$, par l'intermédiaire d'une "équation d'état" que nous ne préciserons pas. Nous n'introduirons pas non plus ici l'entropie $S$, ni la température absolue $T$, pour éviter d'avoir à faire choix d'une équation thermodynamique: nous verrons qu'on peut écrire un système complet d'équations où elles n'interviennent pas explicitement.

$q^{\alpha}$ est le vecteur courant de chaleur. Nous le prendrons de la forme générale ${ }^{3}$ :

$$
q^{\alpha}=q^{\alpha}\left(g^{\lambda \mu}, u^{\beta}, p, f, \partial_{\lambda} p, \partial_{\lambda} f\right) .
$$

$q^{\alpha}$ est une fonction donnée, arbitraire a priori, de ses arguments. Quan

$\tau_{\alpha \beta}$ est le tenseur de Maxwell et a l'expression donnée par Pham Mau

$$
\tau_{\alpha \beta}=\frac{1}{4} g_{\alpha \beta} G^{\lambda \mu} H_{\lambda \mu}-G_{\alpha}^{e} H_{e \beta} .
$$

Selon le point de vue de Pichon [5] nous ne le symétriserons pas.

$H_{\alpha \beta}$ et $G_{\alpha \beta}$ sont respectivement les tenseurs antisymétriques champ électrique-induction magnétique et champ magnétique-induction électrique. Ils définissent deux 2 -formes extérieures

$$
H=\frac{1}{2} H_{\alpha \beta} d x^{\alpha} \wedge d x^{\beta}, G=\frac{1}{2} G_{\alpha \beta} d x^{\alpha} \wedge d x^{\beta} .
$$

Ces tenseurs définissent d'autre part les vecteurs champs (respectivement inductions) électrique et magnétique $e^{\alpha}$ et $h^{\alpha}$ (respectivement $d^{\alpha}$ et $\left.b^{\alpha}\right)$ par les relations ${ }^{4}$

$$
\begin{gathered}
e^{\alpha}=H^{\alpha \beta} u_{\beta}, \quad d^{\alpha}=G^{\alpha \beta} u_{\beta} \\
h^{\alpha}=G^{* \alpha \beta} u_{\beta}, \quad b^{\alpha}=H^{* \alpha \beta} u_{\beta} .
\end{gathered}
$$

Ces vecteurs sont orthogonaux au vecteur vitesse $u^{\alpha}$

$$
h^{\alpha} u_{\alpha}=e^{\alpha} u_{\alpha}=b^{\alpha} u_{\alpha}=d^{\alpha} u_{\alpha}=0 .
$$

Les lois de l'induction de Maxwell, que nous adopterons ici:

$$
b^{\alpha}=\mu h^{\alpha}, \quad d^{\alpha}=\varepsilon e^{\alpha}
$$

${ }^{3}$ Une expression particulière de ce type est donnée par LANDAU-LIFSHITZ [15]. $\partial_{\lambda}$ désigne la dérivié partielle $\partial / \partial x^{\lambda}$.

4 Pour avoir une chaleur de Joule $>0$ avec une conductivité $\sigma>0$ j'ai été amenée à changer le signe dans la définition de $e^{\alpha}$ des travaux antérieurs [16], [2], [3] (c'est-á-dire le signe dans la définition du courant). 
se traduisent par la relation suivante

On a posé:

$$
\mu G_{\alpha \beta}=H_{\alpha \beta}+\lambda u^{\gamma}\left(H_{\gamma \alpha} u_{\beta}-H_{\gamma \beta} u_{\alpha}\right) \text {. }
$$

$$
\lambda=1-\varepsilon \mu .
$$

Les coefficients $\mu$ et $\varepsilon$ sont respectivement la perméabilité magnétique et la permittivité électrique du fluide et ne dépendent que des grandeurs d'état.

Les tenseurs $H_{\alpha \beta}$ et $G_{\alpha \beta}$ vérifient les équations de Maxwell

$$
\begin{aligned}
d H & =0 \\
\nabla_{\alpha} G^{\alpha \beta} & =J^{\beta},
\end{aligned}
$$

où $J^{\beta}$ est le vecteur courant électrique. On prend

$$
J^{\beta}=\gamma u^{\beta}+\sigma e^{\beta}+\beta P^{\beta} .
$$

$\gamma$ est la densité de charge électrique, $\sigma$ la conductivité électrique, $\beta$ un coefficient rendant compte de l'effet Hall (cf. [17], [5]): $\sigma$ et $\beta$ sont des fonctions données des grandeurs d'état. $P^{\beta}$ est le vecteur de Poynting

$$
\mu P_{\alpha}=H^{\sigma \varrho} H_{\varrho \alpha} u_{\sigma}=\mu \varepsilon_{\alpha \lambda \mu \nu} u^{\lambda} e^{\mu} h^{\nu} \text {. }
$$

$J^{\beta}$ satisfait la condition de conservation

$$
\nabla_{\beta} J^{\beta}=0 \text {. }
$$

Nous prendrons d'autre part pour équations déterminant l'évolution du fluide les 5 conditions de conservation:

II

$$
\begin{aligned}
\nabla_{\alpha} T^{\alpha \beta} & =0 \\
\nabla_{\alpha}\left(r u^{\alpha}\right) & =0 .
\end{aligned}
$$

L'équation (1.16), que nous appellerons II, proposée par TAUB, exprime la conservation de la matière.

\section{Systeme équivalent bien posé}

Les équations de Maxwell (1.11) admettent localement pour solution générale ( $\varphi$ désigne une 1 -forme quelconque)

$$
H=d \varphi, \quad \text { c'est-à-dire } H_{\alpha \beta}=\partial_{\alpha} \varphi_{\beta}-\partial_{\beta} \varphi_{\alpha} .
$$

Nous remplacerons les équations de Maxwell 1.12 par

$$
\bar{E}^{\beta} \equiv \nabla_{\alpha} G^{\alpha \beta}-\frac{1}{\mu} g^{\beta \varrho} \nabla_{\varrho}(\delta \varphi)-J^{\beta}=0
$$

où $G^{\alpha \beta}$ s'exprime en fonction de $H^{\alpha \beta}$ par (1.9) et où

$$
\delta \varphi=-\nabla_{\alpha} \varphi^{\alpha}
$$

est le premier membre de la condition de normalisation de Lorentz:

$$
\delta \varphi=0
$$


de manière à mettre en évidence, pour les $\varphi_{\alpha}$, un système d'équations bien posé.

Ces équations (2.2) (que nous appellerons IV) s'écrivent:

$$
\begin{aligned}
\bar{E}_{\beta} & =\frac{1}{\mu} g^{\alpha \lambda}\left\{\nabla_{\alpha} \nabla_{\lambda} \varphi_{\beta}+\lambda u^{\gamma} u_{\beta} \nabla_{\alpha}\left(\nabla_{\gamma} \varphi_{\lambda}-\nabla_{\lambda} \varphi_{\gamma}\right)-\right. \\
\mathrm{IV} \quad & -\lambda u^{\gamma} u_{\lambda} \nabla_{\alpha}\left(\nabla_{\gamma} \varphi_{\beta}-\nabla_{\beta} \varphi_{\gamma}\right)+\lambda H_{\gamma \lambda} \nabla_{\alpha}\left(u^{\gamma} u_{\beta}\right) \\
& \left.-\lambda H_{\gamma \beta} \nabla_{\alpha}\left(u^{\gamma} u_{\lambda}\right)\right\}+R_{\beta}^{\mu} \varphi_{\mu}+u^{\gamma}\left(H_{\gamma}^{\alpha} u_{\beta}-H_{\gamma \beta} u^{\alpha}\right) \partial_{\alpha} \lambda+ \\
& +H_{\beta}^{\alpha} \partial_{\alpha}\left(\mu^{-1}\right)-J_{\beta}=0 .
\end{aligned}
$$

D'autre part, toute solution de (1.15), (1.3), (1.16) vérifie l'équation de continuité, soit III:

III

$$
u_{\beta} \nabla_{\alpha} T^{\alpha \beta}=u^{\alpha}\left(r \partial_{\alpha} f-\partial_{\alpha} p\right)+u_{\beta} \nabla_{\alpha} \tau^{\alpha \beta}-\nabla_{\alpha} q^{\alpha}=0
$$

avec

$$
u_{\beta} \nabla_{\alpha} \tau^{\alpha \beta}=-\sigma|e|^{2}+\left.(1-\varepsilon \mu) P^{\alpha} u^{\beta} \nabla_{\beta} u_{\alpha}|,| e\right|^{2}=-e^{\lambda} e_{\lambda}
$$

(qui, pour $\varepsilon=\mu=1$, se réduit, si on pose $q^{\alpha}=-\chi \gamma^{\alpha \beta} \partial_{\beta} T$ (cf. [2]), à la formulation quadridimensionnelle de l'équation classique de la chaleur dans les gaz) et les équations du mouvement, soit I

$$
\begin{gathered}
\gamma_{\lambda}^{\beta} \nabla_{\alpha} T^{\alpha \lambda}=\left(r f u^{\alpha}-q^{\alpha}\right) \nabla_{\alpha} u^{\beta}-\gamma^{\alpha \beta} \partial_{\alpha} p+\gamma_{\lambda}^{\beta} \nabla_{\alpha} \tau^{\alpha \lambda}=0 \\
\gamma_{\alpha}^{\beta}=g_{\alpha}^{\beta}-u_{\alpha} u^{\beta} \quad \text { (projecteur d'espace) }
\end{gathered}
$$

avec (cf. [2], [5])

$$
\nabla_{\alpha} \tau^{\alpha \beta}=J^{\lambda} H_{\lambda}^{\beta}+\lambda P_{\alpha} \nabla^{\beta} u^{\alpha}+\frac{1}{2} g^{\beta \lambda}\left(e^{\sigma} e_{\sigma} \partial_{\lambda} \varepsilon+h^{\sigma} h_{\sigma} \partial_{\lambda} \mu\right)
$$

où, d'après les hypothèses,

$$
\begin{gathered}
\partial_{\lambda} \varepsilon=\varepsilon_{p}^{\prime} \partial_{\lambda} p+\varepsilon_{f}^{\prime} \partial_{\lambda} f \\
\partial_{\lambda} \mu=\mu_{p}^{\prime} \partial_{\lambda} p+\mu_{f}^{\prime} \partial_{\lambda} f^{\prime} \quad\left(\varepsilon_{p}^{\prime}=\frac{\partial \varepsilon}{\partial p}, \text { etc. }\right)
\end{gathered}
$$

(le dernier terme de la force de Lorenz (2.6) provient des phénomènes dits électrostriction et magnétostriction $)^{5}$

on posera

$$
\begin{aligned}
& \frac{1}{2}\left(e^{\sigma} e_{\sigma} \varepsilon_{p}^{\prime}+h^{\sigma} h_{\sigma} \mu_{p}^{\prime}\right)=b \\
& \frac{1}{2}\left(e^{\sigma} e_{\sigma} \varepsilon_{f}^{\prime}+h^{\sigma} h_{\sigma} \mu_{f}^{\prime}\right)=b^{\prime} .
\end{aligned}
$$

\section{Etude des equations obtenues}

Nous avons remplacé les équations satisfaites par les grandeurs physiques, inconnues du problème posé, $u^{\alpha}, f, p, \varphi_{\alpha}, \gamma$ par le système soit $(\Sigma)$, d'équations I, II, III, IV (cf. paragraphe 2) et V (cf. paragraphe 1).

5 Ajouter des termes en dérivées de $\varepsilon$ et $\mu$ au tenseur de Maxwell (cf. [18], [5], [19]) rendrait impossible, à cause des ordres de dérivation, l'étude qui va suivre: il semble d'ailleurs que, physiquement ces termes soient assez mal connus. 
Nous montrerons plus loin (paragraphe 19) que ce système $(\Sigma)$ est équivalent au système tensoriel donné physiquement (équations de Maxwell, équations de conservation (1.15) et (1.16), $u^{\alpha}$ unitaire). Nous allons d'abord, ici, chercher à quelles conditions ce système $(\Sigma)$ est un système d'équations aux dérivées partielles bien posé au sens de la physique mathématique, c'est-à-dire un système quasi-linéaire du type de CauchyKovalevski: pour un tel système le problème de Cauchy analytique a une solution et une seule si la variété (de dimensions 3) portant les données initiales n'est pas exceptionnelle (relativement à ces données initiales, cas non linéaire). Les variétés exceptionnelles sont dites "caractéristiques».

Désignons par $v^{I}$ une quelconque des $N$ inconnues $u^{\alpha}, f, p, \varphi_{\alpha}, \gamma$, par $E^{J}$ une quelconque des $N$ équations du système $(\Sigma)$. Le système $(\Sigma)$ est quasi-linéaire (cf. LeRAY [20]) si on peut trouver des indices $m_{I}$ et $n_{J}$ tels qu'il s'écrive

$$
E^{J}=h_{I}^{J}\left(D^{m_{K}-n_{J}-1} v^{K}, \tilde{D}^{m_{I}-n_{J}}\right) v^{I}+b^{J}\left(D^{m_{K}-n_{J}-1} v^{K}\right)=0
$$

où $D^{m}$ désigne l'ensemble des dérivations partielles d'ordre $\leqq m, \tilde{D}^{m}$ celles d'ordre $m$. L'opérateur $h_{I}^{J}$ d'ordre $m_{I}-n_{J}$, qui peut être nul, est appelé la partie principale de l'inconnue $v^{I}$ dans l'équation $E^{J}$.

On voit aisément que pour que le système $(\Sigma)$ soit quasilinéaire ${ }^{6}$ il faut que $q^{\alpha}$ ne dépende des dérivées des grandeurs d'état que par l'intermédiaire du gradient d'une fonction $\Phi$ des ces grandeurs, qui peut par exemple (mais pas nécessairement) être la température absolue:

$$
q^{\alpha}=q^{\alpha}\left(u^{\beta}, g^{\lambda \mu}, p, f, \partial_{\lambda} \Phi\right), \quad \Phi \equiv \Phi(p, f) .
$$

On supposera donc $q^{\alpha}$ de cette forme et on prendra $\Phi$ comme inconnue à la place par exemple de $f$. Le système $(\Sigma)$ sera alors quasi linéaire pour le choix suivant des indices de Leray:

$$
\begin{aligned}
& m\left(u^{\alpha}\right)=m(p)=m(\gamma)=1 \\
& m\left(\varphi_{\alpha}\right)=m(\Phi)=2 \\
& n(\mathrm{I})=n(\mathrm{II})=\cdots=n(\mathrm{~V})=0 .
\end{aligned}
$$

Les parties principales relativement aux inconnues $u^{\alpha}, f, \Phi, \varphi_{\alpha}, \gamma$ dans toutes les équations sont alors respectivement d'ordre $1,1,2,2,1$.

Le système $(\Sigma)$ est du type de Cauchy-Kovalevski (généralisé par LERAY-GARDING) si la partie principale, d'ordre $\Sigma_{K} m_{K}-\Sigma_{J} n_{J}=16 \mathrm{du}$ déterminant $h$ des $h_{I}^{J}$, défini par ( $\pi$ est une permutation quelconque des entiers $1, \ldots N)$ :

$$
h=\Sigma_{\pi} \operatorname{Sign} \pi h_{\pi(1)}^{1} \ldots h_{\pi(N)}^{N}
$$

n'est pas nulle. C'est-à-dire si le polynôme homogène de degré $16, P$, n'est pas identiquement nul:

$$
P=\operatorname{det}\left(P_{I}^{J}\right) \neq 0
$$

6 Et á déterminant caractéristique non nul (cf. plus loin). 
où $P_{I}^{J}$ est le polynôme homogène en $\xi_{\alpha}$ obtenu en remplaçant dans $h_{I}^{J}$ la dérivation $\partial_{\alpha}$ par le vecteur covariant $\xi_{\alpha}$.

Nous allons vérifier (3.4) en même temps que nous allons déterminer les variétés caractéristiques.

\section{Variétés caractéristiques}

Les vecteurs $\xi_{\alpha}$ qui annulent en un point $x$ le polynôme $P$ (non $\equiv 0$, ce que nous allons vérifier) sont les normales en ce point aux variétés caractéristiques, ou, fronts d'onde (qui dépendent ici des inconnues $v^{I}$ ).

Le polynôme $P$ est un déterminant d'éléments $P_{I}^{J}$. Les parties principales relatives aux inconnues $\varphi_{\alpha}$ étant nulles dans les équations I, II, III et celles relatives à $\Phi$ nulles dans toutes les équations sauf III, ce déterminant est du type suivant:

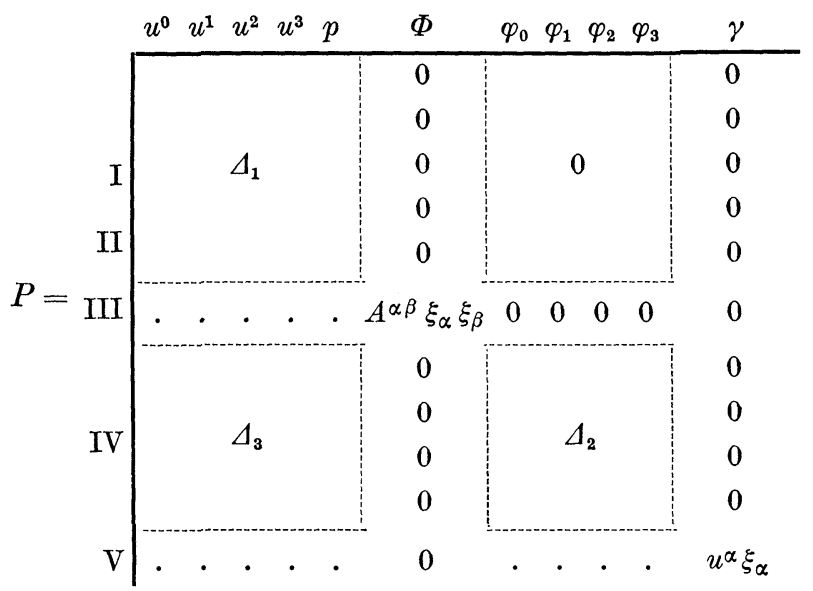

où $\Delta_{1}$ est le déterminant d'ordre 5 d'éléments

$$
h_{\alpha}^{\beta}=\left(r f u^{\lambda}-q^{\lambda}\right) \xi_{\lambda} \delta_{\alpha}^{\beta}+\lambda \gamma^{\beta \gamma} \xi_{\gamma} P_{\alpha}, \quad \alpha=0,1,2,3
$$

(tenseur mixte sur $V_{4}$ )

$$
h_{\alpha}^{4}=r \xi_{\alpha}, \quad h_{4}^{\alpha}=\gamma^{\alpha \beta} \xi_{\beta}(b-1)
$$

(vecteurs respectivement covariants et contrevariants sur $V_{4}$ )

$$
h_{4}^{4}=r_{p}^{\prime} u^{\alpha} \xi_{\alpha}
$$

(scalaire sur $V_{4}$ ) et où $\Delta_{2}$ est le déterminant d'ordre 4 (ici l'indice inférieur numérote la ligne et l'indice supérieur la colonne contrairement au déterminant $\Delta_{1}$ )

$$
h_{\bar{\beta}}^{\bar{\alpha}}=\frac{1}{\mu}\left(g-\lambda a^{2}\right) \delta_{\bar{\beta}}^{\bar{\alpha}}+\lambda u_{\bar{\beta}}\left(a \xi^{\bar{\alpha}}-g u^{\bar{\alpha}}\right)+\lambda a \xi_{\bar{\beta}} u^{\bar{\alpha}},
$$

tenseur mixte sur $V_{4}$; on a posé:

$$
a=u^{\alpha} \xi_{\alpha}, \quad g=g^{\alpha \beta} \xi_{\alpha} \xi_{\beta} .
$$


On voit sur l'expression (4.1) que $P$ est $\neq 0$ :

$$
P=\left(u^{\alpha} \xi_{\alpha}\right)\left(A^{\lambda \mu} \xi_{\lambda} \xi_{\mu}\right) \Delta_{1} \Delta_{2} .
$$

Les déterminants $\Delta_{1}$ et $\Delta_{2}$ sont des scalaires que l'on peut calculer aisément en prenant des axes particuliers (corepère $\theta^{0}$ colinéaire à $\xi_{\alpha}$, corepère $\theta^{1}$ colinéaire respectivement à $P_{\alpha}$ ou à $u_{\alpha}$ pour le calcul de $\Delta_{1}$ ou de $\left.\Delta_{2}\right)$. On trouve

$$
\Delta_{1}=\left\{\left(r f u^{\alpha}-q^{\alpha}\right) \xi_{\alpha}\right\}^{3} r C_{1}(\xi)
$$

où $C_{1}(\xi)$ est le polynôme du $2^{\text {ème }}$ degré

$$
\begin{aligned}
C_{1}(\xi)=\gamma^{\alpha \beta} \xi_{\alpha} \xi_{\beta}+u^{\alpha} u^{\beta}\left(f r_{p}^{\prime}+\frac{1}{2}\left(\lambda_{p}^{\prime}|e|^{2}\right.\right. & \left.+\frac{1}{2} \mu_{p}^{\prime}|h|^{2}\right) \xi_{\alpha} \xi_{\beta}+ \\
& +\left(\lambda P^{\alpha}-q^{\alpha}\right) u^{\beta} \xi_{\alpha} \xi_{\beta} r_{p}^{\prime} r^{-1}
\end{aligned}
$$

et

$$
\Delta_{2}=\left(C_{2}(\xi)\right)^{2}\left(C_{3}(\xi)\right)^{2}
$$

où $C_{2}(\xi)$ et $C_{3}(\xi)$ sont les polynômes caractéristiques classiques des fluides inductifs (cf. [2], [5]):

$$
\begin{aligned}
& C_{2}(\xi)=g^{\lambda \mu} \xi_{\lambda} \xi_{\mu} \\
& C_{3}(\xi)=\left(g^{\lambda \mu}-\lambda u^{\lambda} u^{\mu}\right) \xi_{\lambda} \xi_{\mu} .
\end{aligned}
$$

Le polynôme $A^{\alpha \beta} \xi_{\alpha} \xi_{\beta}$ est d'autre part:

$$
C_{4}(\xi)=A^{\alpha \beta} \xi_{\alpha} \xi_{\beta}=\frac{\partial q^{\alpha}}{\partial\left(\partial_{\beta} \Phi\right)} \xi_{\alpha} \xi_{\beta}
$$

et dépend du choix fait pour les fonction $q^{\alpha}$. En s'inspirant de la théorie classique de la chaleur de Fourier, et identifiant $\Phi$ avec la température absolue $T$, Pham Mau Quan a proposé ( $\chi$ conductibilité thermique):

Done

$$
q^{\alpha}=-\chi \gamma^{\alpha \beta} \partial_{\beta} T
$$

$$
A^{\alpha \beta} \xi_{\alpha} \xi_{\beta}=-\chi \gamma^{\alpha \beta} \xi_{\alpha} \xi_{\beta} \text {. }
$$

Nous reviendrons paragraphe 5 sur la discussion de ce choix.

\section{Ondes-vitesses de propagation}

L'annulation de chaque facteur irréductible du polynôme $P$, où $\xi_{\alpha}$ est remplacé à nouveau par $\partial_{\alpha}$, donne une équation aux dérivées partielles $d u$ premier ordre dont les solutions forment une famille de variétés caractéristiques (ou fronts d'ondes) du système $(\Sigma)$ étudié. Les pentes spatiotemporelles, dans un repère orthonormé, du plan tangent à une telle variété en un point, (plan normal au cône) sont les vitesses de propagation en ce point des fronts d'ondes correspondants: ces vitesses seront inférieures à la vitesse relativiste limite si les cônes $C(\xi)=0$ (où les $C(\xi)$ sont les facteurs irréductibles de $P$ ) contiennent dans leur intérieur le cône fondamental de la métrique, $C_{2}(\xi)=0$. 
Cônes caractéristiques. Pour étudier ces divers cônes en un point, plaçons-nous dans un repère propre: $g^{\alpha \beta} \xi_{\alpha} \xi_{\beta} \equiv\left(\xi_{\alpha}\right)^{2}-\Sigma_{i}\left(\xi_{i}\right)^{2}, u^{0}=1$, $u^{i}=0$. Allors $P^{0}=0$ car $P^{\alpha}$ et $u^{\alpha}$ sont orthogonaux (et aussi $q^{0}=0$ sous l'hypothèse (4.13)). Dans ce repère les divers cônes s'écrivent de la facon suivante:

a) Cône gravitationnel ou fondamental

$$
C_{2}(\xi) \equiv\left(\xi_{0}\right)^{2}-\Sigma_{i}\left(\xi_{i}\right)^{2}=0 .
$$

b) Cône électromagnétique

$$
C_{3}(\xi) \equiv \varepsilon \mu\left(\xi_{0}\right)^{2}-\Sigma_{i}\left(\xi_{i}\right)^{2}=0 .
$$

c) Cône (plan) matériel

$$
C_{0}(\xi) \equiv u^{\alpha} \xi_{\alpha}=\xi_{0}=0 .
$$

d) Cône (plan) énergétique

$$
C_{0}^{\prime}(\xi) \equiv\left(r f u^{\alpha}-q^{\alpha}\right) \xi_{\alpha}=\left(r f-q^{0}\right) \xi_{0}-\Sigma_{i} q^{i} \xi_{i}=0 .
$$

e) Cône énergétique

$$
C_{4}(\xi) \equiv A^{\alpha \beta} \xi_{\alpha} \xi_{\beta}=0 .
$$

Sous les hypothèses 4.13 et 4.14

f) Cône acoustique

$$
C_{4}(\xi) \equiv \Sigma_{i}\left(\xi_{i}\right)^{2}=0 .
$$

$$
\begin{aligned}
C_{1}(\xi)=\left\{\left(f-q^{0} r^{-1}\right) r_{p}^{\prime}-\frac{1}{2} \lambda_{p}^{\prime}|e|^{2}-\frac{1}{2} \mu_{p}^{\prime}|h|^{2}\right\}\left(\xi_{0}\right)^{2}+ \\
+r_{p}^{\prime} r^{-1}\left(\lambda P^{i}-q^{i}\right) \xi_{i} \xi_{0}-\Sigma_{i}\left(\xi_{i}\right)^{2}=0 .
\end{aligned}
$$

Plaçons ces divers cônes par rapport au cône fondamental $C_{2}(\xi)$. à $C_{3}$

a) Si $\varepsilon \mu>1$ (cas physique) le cône $C_{3}$ est convexe et $C_{2}$ est intérieur

b) le plan $C_{0}$ est extérieur à $C_{2}$ (si $u$ est temporel)

c) le plan $C_{0}^{\prime}$ est extérieur à $C_{2}$ si

$$
\frac{|\pi \mathbf{q}|}{r f-\mathbf{u q}}<1
$$

où $\pi q$ désigne la projection de $q$ sur l'espace propre orthogonal à $\mathbf{u}$. On a $\pi q=q$ sous l'hypothèse (4.13), $q u=0$. (5.8) sera toujours réalisé sous les conditions physiques usuelles: $|q|$ petit devant $r f$.

d) Le cône $C_{4}$ est, sous les hypothèses (4.13), (4.14) un cylindre de génératrices parallèles à $\mathbf{u}$ de base le cône isotrope de l'espace propre, il admet donc une seule génératrice réelle, de direction $\mathbf{u}$, et $C_{2}$ n'est pas intérieur à $C_{4}$.

e) Le cône acoustique $C_{1}$ est convexe, et le vecteur $\mathbf{u}=\mathrm{e}_{0}$ lui est intérieur si le coefficient de $\left(\xi_{0}\right)^{2}$ est $>0$, ce qui est toujours réalisé physiquement $\left(r f \gg q^{0}\right) . C_{2}$ n'est intérieur à $C_{1}$ que si un certain nombre 
d'inégalités (que l'on pourrait considérer comme caractérisant les fluides réels, le cas limite correspondant aux fluides incompressibles) sont vérifiées. Nous discuterons ces inégalités en détail paragraphe 8 , quand le courant de chaleur est nul.

Vitesses de propagation. Des raisonnements classiques ([16], [3], [5]) permettent de déduire des équations précédentes les vitesses de propagation des ondes correspondantes par rapport au repère propre (ces vitesses dépendent de la direction de propagation $n$ pour les ondes acoustiques et énergétiques). Ce sont:

a) Ondes gravitationnelles (ou fondamentales)

$$
v=1 \text {. }
$$

b) Ondes électromagnétiques

$$
v=\frac{1}{(\varepsilon \mu)^{1 / 2}} \leqq 1
$$

c) Ondes matérielles

d) Ondes énergétiques

$$
v=0 .
$$

$$
(v)^{2}=\frac{\pi \mathbf{q} \cdot \mathbf{n}}{r f-\mathbf{u} \cdot \mathbf{q}}
$$

où $\pi q$ est la projection sur l'espace propre (orthogonal à $u$ ) de q. On a toujours

$$
(v)^{2} \ll 1 \text {. }
$$

e) Ondes calorifiques.

L'hypothèse (4.14) conduirait à une vitesse de propagation infinie: cette hypothèse semble donc à rejeter.

f) Ondes acoustiques.

Les vitesses de propagation $v$ dans la direction $n$ dépendent du vecteur de Poynting et du courant de chaleur, étant solutions de l'équation du second degré:

$$
\begin{gathered}
\left(\left(f-q^{0} r^{-1}\right) r_{p}^{\prime}-\frac{1}{2} \varepsilon_{p}^{\prime}|e|^{2}-\frac{1}{2} \mu_{p}^{\prime}|h|^{2}\right) v^{2}+ \\
+r_{p}^{\prime} r^{-1}(\lambda \mathbf{p}-\mathbf{q}) \cdot \mathbf{n} v-1=0 .
\end{gathered}
$$

Ces vitesses sont inférieures à 1 sous la condition signalée plus haut $\left(C_{2}\right.$ intérieur à $\left.C_{1}\right)$.

\section{Cas de la conductibilité thermique nulle}

\section{Polynôme caractéristique}

Dire que la conductibilité thermique est nulle, c'est dire que le vecteur courant de chaleur $q^{\alpha}$ est nul. L'équation III est alors du premier degré dans toutes les grandeurs d'état. 
Pour écrire le système $(\Sigma)$ sous la forme 3.1 on peut poser

$$
\begin{aligned}
m\left(u^{\alpha}\right) & =m(p)=m(f)=m(\gamma)=1 \\
m\left(\varphi_{\alpha}\right) & =2 \\
n_{\mathrm{I}} & =0, \quad I=1, \ldots N .
\end{aligned}
$$

Par un raisonnement analogue à celui du paragraphe précédent on voit que le déterminant caractéristique $P$ est alors

$$
P=u^{\alpha} \xi_{\alpha} \Delta_{1}^{\prime} \Delta_{2}
$$

où $\Delta_{1}^{\prime}$ est le déterminant d'ordre 6 obtenu en bordant $\Delta_{1}$ (cf. paragraphe 4 ) par la colonne et la ligne suivantes: ( $b$ et $b^{\prime}$ sont définis par (2.7))

$$
h_{5}^{\alpha}=-\gamma^{\alpha \beta} \xi_{\beta} b^{\prime}, \quad \alpha=0,1,2,3
$$

(vecteur contrevariant)

(scalaires)

$$
\begin{aligned}
& h_{5}^{4}=r_{f}^{\prime} u^{\alpha} \xi_{\alpha} \\
& h_{5}^{5}=\left(r+b^{\prime}\right) u^{\alpha} \xi_{\alpha} \\
& h_{4}^{5}=-b u^{\alpha} \xi_{\alpha}
\end{aligned}
$$

(vecteur covariant).

$$
h_{\alpha}^{5}=\lambda P_{\alpha} u^{\beta} \xi_{\beta}
$$

Le même repère que précédemment permet de calculer le scalaire $\Delta_{1}^{\prime}$

$$
\Delta_{1}^{\prime}=r^{2} f\left(u^{\alpha} \xi_{\alpha}\right)^{4} C_{1}^{\prime}(\xi)
$$

où $C_{1}^{\prime}(\xi)$ est le polynôme du second degré

$$
\begin{array}{r}
C_{1}^{\prime}(\xi)=r(1-b) \gamma^{\alpha \beta} \xi_{\alpha} \xi_{\beta}+f\left(r_{p}^{\prime}\left(r+b^{\prime}\right)+r_{f}^{\prime}(1-b)\right)\left(u^{\alpha} \xi_{\alpha}\right)^{2}+ \\
+\lambda P^{\alpha} u^{\beta} \xi_{\alpha} \xi_{\beta} r_{p}^{\prime} .
\end{array}
$$

Alors que l'on a toujours

$$
\Delta_{2}=\left(C_{2}(\xi)\right)^{2}\left(C_{3}(\xi)\right)^{2} .
$$

\section{Cas de G. Pichon}

Dans le cas où l'équation d'état est telle que $\varrho=r(1+\mathscr{E})=r f-p$ ne dépend que de $p$ (et non de $f$ ); on a:

$$
\begin{aligned}
& \varrho_{f}^{\prime}=f r_{f}^{\prime}+r=0 \\
& \varrho_{p}^{\prime}=f r_{p}^{\prime}-1 .
\end{aligned}
$$

Done

$$
f r_{f}^{\prime}+f r r_{p}^{\prime}=r \varrho_{p}^{\prime}
$$

On retrouve si $b=b^{\prime}=0$ (c'est-à-dire $\varepsilon$ et $\mu$ constants) les résultats de Pichon [5]. 


\section{Fronts d'ondes et vitesses de propagation}

Comparons les résultats obtenus avec ceux de la partie I: on voit que le cône énergétique est confondu avec le cône (plan) matériel, le cône calorifique disparaît, le cône électromagnétique $C_{3}$ est inchangé. Le cône acoustique $C_{1}$ devient le cône $C_{1}^{\prime}$ qui, dans un repère propre a pour équation:

$$
\begin{aligned}
C_{1}^{\prime}(\xi)=f\left(r_{p}^{\prime}\left(r+b^{\prime}\right)+r_{f}^{\prime}(1-b)\right) & \left(\xi_{0}\right)^{2}+ \\
& +\lambda r_{p}^{\prime} P^{i} \xi_{i} \xi_{0}-r(1-b) \Sigma_{i}\left(\xi_{i}\right)^{2}=0
\end{aligned}
$$

cône convexe réel si

$$
A \equiv f\left(r_{p}^{\prime}\left(r+b^{\prime}\right)+r_{f}^{\prime}(1-b)\right)>0, \quad r(1-b)>0
$$

ce qui sera toujours réalisé physiquement $\left(r>0, f>0, r_{p}^{\prime}>0, r_{f}^{\prime}>0, b\right.$ et $b^{\prime}$ très petits).

Le cône $C_{1}^{\prime}(\xi)=0$ ne coupe alors pas le plan $\xi_{0}=0$ (c'est-à-dire le plan $u^{a} \xi_{a}=0$ ).

Etudions la position de $C_{1}^{\prime}(\xi)=0$ par rapport au cône fondamental $C_{2}(\xi)=0$. Les cônes $C_{1}^{\prime}$ et $C_{2}$ ne se coupent pas si

$$
|A-r(1-b)| \geqq\left|\lambda r_{p}^{\prime} P\right| \text {. }
$$

$C_{2}$ est alors intérieur à $C_{1}$ si

$$
A \geqq r(1-b),
$$

extérieur dans le cas contraire.

Si l'inégalité (8.3) n'est pas vérifiée, $C_{1}^{\prime}$ et $C_{2}$ se coupent suivant deux droites.

L'équation d'état du fluide relativiste devrait donc être telle que (8.3) et (8.4) soient vérifiées. Si $b=b^{\prime}=0$ (8.3) s'écrit, en supposant $\varepsilon \mu \geqq 1$, $\varrho_{p}^{\prime}$ et $r_{p}^{\prime}>0$

$$
\left(\varrho_{p}^{\prime}-1\right)+r^{-1} \varrho_{f}^{\prime} \geqq \frac{(\varepsilon \mu-1)}{r f}\left(\varrho_{p}^{\prime}+1\right)|P|
$$

qui entraîne alors

$$
\varrho_{p}^{\prime}-1+r^{-1} \varrho_{f}^{\prime} \geqq 0 \text {. }
$$

Les inégalités (8.3) et (8.4) se réduisent à (8.6) en l'absence d'inductions $(\varepsilon=\mu=1)$ : dans ce cas on prend l'égalité

$$
\varrho_{p}^{\prime}-1+r^{-1} \varrho_{f}^{\prime}=0
$$

comme définition de l'incompressibilité du fluide (cf. [11]) et on suppose que tout fluide réel (non inductif) vérifie (8.6), donc que le cône fondamental est bien intérieur au cône acoustique.

Dans le cas inductif il faudrait, pour rester en accord avec les principes relativistes, prendre une définition de l'incompressibilité telle que tout fluide réel vérifie (8.3), (8.4); sous les conditions physiques pratiquement toujours vérifiées (cf. (8.2)), ces deux inégalités se réduisent à la suivante:

$$
A \geqq r(1-b)+\lambda r_{p}^{\prime}|P| \text {. }
$$


Si les fonctions $v^{I}\left(u^{a}, f, p, \varphi_{\lambda}, \gamma\right)$ vérifient cette inégalité le cône $C_{1}^{\prime}$ admet comme les autres cônes $C_{i}, C_{2}$ dans son intérieur.

\section{Caractère hyperbolique non strict du système}

Définition et critère d'hyperbolicité non stricte: le système quasi linéaire

$$
h_{I}^{J}\left(D^{m_{K}-n_{J}-1} u^{K}, D^{m_{I}-n_{J}}\right) u^{I}+b^{J}\left(D^{m_{K}-n_{J}-1} u_{K}\right)=0
$$

est hyperbolique non strict au sens de Leray-Ohya ${ }^{7}$ pour des fonctions $W^{K}\left(C^{\infty}\right)$ si (condition suffisante) en faisant $D^{m_{K-n}-1} u^{K}=D^{m_{K}-n_{J}-1} W^{K}$, $J, K=1, \ldots, N$ dans les coefficients de (9.1) les propriétés suivantes sont vérifiées:

1) Le polynôme caractéristique $P$ est un produit de polynômes hyperboliques stricts $^{8}$

$$
P=P_{1}(\xi) \ldots P_{m}(\xi) .
$$

2) Les cônes $P_{i}(\xi)=0$ contiennent tous dans leur intérieur un même cône, celui-ci détermine alors le »domaine d'influence« de la solution.

$\left(l_{i}=\right.$ degré du polynôme $P_{i}$ ).

3) $\operatorname{Sup} l_{i} \geqq \operatorname{Sup}_{K} m_{K}-\inf _{J} n_{J}$

Les conditions 1$), 2), 3$ ) sont réalisées pour le système $(\Sigma)$, et des fonctions $W^{K}$ vérifiant les inégalités énoncées au paragraphe précédent: le polynôme $P(\xi)$ est évidemment produit de polynômes hyperboliques (de degrés 1 ou 2 ), et les cônes $C_{i}(\xi)=0$ contiennent tous dans leur intérieur le cône fondamental $C_{2}$.

(9.3) est vérifié puisque $\operatorname{Sup} l_{i}=2, \operatorname{Sup} m_{K}-\inf n_{J}=2$.

Problème de Cauchy. Les données de Cauchy, sur une variété initiale $S$ sont:

$$
D^{m_{K}-1} u^{K}=D^{m_{K}-1} W^{K} \quad \text { sur } \quad S
$$

où les $W^{K}$ sont des fonctions données dans un voisinage de $S$. Si $S$ a pour équation locale $x^{0}=0(9.4)$ est équivalent à se donner (on pose $D_{0}=\partial / \partial x^{0}$ )

c'est-à-dire ici

$$
\left\{D_{0}^{m_{K}-1} u^{K}\right\}_{x^{0}=0}
$$

$$
\left\{u^{\alpha}, f, p, \varphi_{\lambda}, \partial_{0} \varphi_{\lambda}, \gamma\right\}_{x^{0}=0} .
$$

Remarque: pour le système $(\Sigma)$ considéré ici on a pu choisir $n_{J}=0$, les données de Cauchy ne sont donc pas soumises à des contraintes ${ }^{9}$.

7 Pour la définition des systèmes diagonaux hyperboliques non stricts, et la démonstration des théorèmes d'existence et d'unicité correspondants, cf. LERAY et OHYA [9]. Pour la diagonalisation des systèmes quasi-linéaires et des critères suffisants d'hyperbolicité non stricte cf. BRUHAT [21].

${ }^{8}$ Un polynôme $P_{i}(\xi)$ de degré $l_{i}$ est hyperbolique strict si le cone $\Gamma_{i}\left(P_{i}(\xi)=0\right)$ a un intérieur non vide $\Gamma_{i}$ tel que toute droite passant par un point de $\Gamma_{i}$ coupe $\Gamma_{\boldsymbol{i}}$ en $l_{i}$ points réels distincts (cf. LERAY [20]).

${ }^{9}$ Il n'en est pas ainsi pour le systéme tensoriel primitif comme on le verra paragraphe 12. 
Pour des données de Cauchy sur $S$ telles qu'il existe des $W^{K}$ correspondant vérifiant les conditions d'hyperbolicité non stricte énoncées ci-dessus (c'est-à-dire telles que dans un voisinage des valeurs prises par ces données les inégalités du paragraphe 8 soient vérifiées), et appartenant à une classe de Gevrey ${ }^{10} \mathrm{~d}$ 'indice $\alpha=\frac{m}{m-1}$, le système $(\Sigma)$ a une solution, unique, dans cette classe de Gevrey. Cette solution admet un domaine d'influence déterminé par le cône $C_{2}$, c'est-à-dire que sa valeur en un point $x$ ne dépend que des données de Cauchy dans le "passé», (au sens relativiste de ce mot, déterminé par le cône $C_{2}$ ) de ce point, sous les inégalités du paragraphe 8.

Calcul de l'indice de Gevrey. L'indice de Gevrey $\alpha, \geqq 1$, caractérise la généralité de la classe de Gevrey envisagée: la classe de Gevrey $\gamma_{2}^{(a)}(S)$ à laquelle doivent appartenir les données de Cauchy (9.5) est l'ensemble des fonctions définies sur $S$, à valeurs complexes, indéfiniment dérivables si $\alpha<\infty$ telles que:

$$
\operatorname{Sup}_{\sigma} \frac{1}{[1+|\sigma|]^{\alpha}}\left(D^{\sigma} f, S\right)<\infty
$$

où $D^{\sigma}$ désigne ici une dérivée partielle quelconque de $f$ sur $S$ (c'est-à-dire par rapport aux variables «spatiales» $\left.x^{i}\right),|\sigma|$ l'ordre de cette dérivée, $\left(D^{\sigma} f, S\right)$ la norme $L_{2}$ usuelle:

$$
\left(D^{\sigma} f, S\right)=\left\{\int_{S}\left|D^{\sigma} f\right|^{2} d x^{1} d x^{2} d x^{3}\right\}^{1 / 2}
$$

Si $\alpha=\infty$ on appelle $\gamma_{2}^{(\infty)}(S)$ l'ensemble des fonctions de carré sommable sur $S$.

On voit qu'une classe de Gevrey d'indice $\alpha$ est incluse dans toutes les classes d'indice plus grand. On montre que pour $\alpha=1$ la classe de Gevrey est une classe de fonctions analytiques et que, par contre, pour $\alpha>1$ ce sont des classes de fonctions non quasi analytiques: une fonction à support compact n'est plus nécessairement nulle.

Physiquement la différence essentielle semble être d'abord entre $\alpha=1$ et $\alpha>1, \alpha>1$ permettant l'existence d'un domaine d'influence donc l'étude de la propagation des phénomènes et caractérisant pour nous l'hyperbolique non strict, puis entre $\alpha<\infty$ et $\alpha=\infty, \alpha=\infty$ permettant l'existence d'un nombre fini seulement de dérivées, donc de discontinuités de celles-ci (chocs) et caractérisant l'hyperbolique strict.

Une première étude simple va nous donner une borne inférieure $\alpha=\frac{m}{m-1}>1$ de l'indice de Gevrey. L'étude du paragraphe suivant, plus complexe, nous permettra d'améliorer cette valeur, sans arriver toutefois à $\alpha=\infty$. Cette étude est cependant utile, non seulement pour

10 Fonctions indéfiniment dérivables dont les dérivées satisfont à certaines majorations ef. LeRAY et OHYA [8], [9]. 
élargir la classe des fonctions pour lesquelles le théorème d'existence est valable, mais aussi pour obtenir les propriétés d'hyperbolicité stricte (ou non) de systèmes partiels que l'on peut avoir à considérer dans des problèmes particuliers.

Ecrivons le polynôme $P(6.2)$ de manière à y faire apparaître le plus petit nombre $m$ possible de polynômes hyperboliques, pour que l'indice $\alpha$ (donc la classe de Gevrey) soit aussi grand que possible. On peut écrire

$$
P=r^{2} f\left(u^{\alpha} \xi_{\alpha}\right)^{5} C_{1}^{\prime}(\xi)\left(C_{2}(\xi)\right)^{2}\left(C_{3}(\xi)\right)^{2} .
$$

D'après l'étude du paragraphe $6,(9.6)$ peut s'écrire:

où

$$
P=P_{1} P_{2} P_{3} P_{4} P_{5}
$$

$$
\begin{aligned}
& P_{1}=r^{2} f u^{\alpha} \xi_{\alpha} \\
& P_{2}=u^{\alpha} \xi_{\alpha} \\
& P_{3}=\left(u^{\alpha} \xi_{\alpha}\right) C_{1}^{\prime}(\xi) \\
& P_{4}=C_{2}(\xi) C_{3}(\xi) u^{\alpha} \xi_{\alpha} \\
& P_{5}=C_{2}(\xi) C_{3}(\xi) u^{\alpha} \xi_{\alpha}
\end{aligned}
$$

où, sous les mêmes conditions que précédemment les polynômes $P_{i}$ sont hyperboliques et les cônes $\Gamma_{i}$ correspondants admettent tous dans leur intérieur le cône $C_{2}$. On obtient donc ainsi l'existence et l'unicité (ainsi que la causalité relativiste) dans une classe de Gevrey d'indice $\alpha=5 / 4$.

\section{Simplification}

Nous avons montré dans [21] que quand tous les mineurs $p_{J}^{I}$ du déterminant d'éléments $P_{I}^{J}$ contiennent en facteur un même polynôme $f(\xi)$ (qui est nécessairement produit de facteurs irréductibles de $P$ ) on peut obtenir une forme diagonale simplifiée pour le système.

Si le polynôme

$$
\hat{P}(\xi)=\frac{P(\xi)}{f(\xi)}
$$

est produit de $m^{\prime}$ facteurs hyperboliques stricts, le système aux dérivées partielles a une solution et une seule dans une classe de Gevrey d'indice

$$
\alpha^{\prime}=\frac{m^{\prime}}{m^{\prime}-1} \text {. }
$$

Etudions cette simplification quand $\varepsilon$ et $\mu$ sont constants ainsi que $\sigma$ et que $\beta$ (coefficient de Hall) est nul. 
Le déterminant $P=\Delta$ s'écrit (cf. paragraphe 6 )

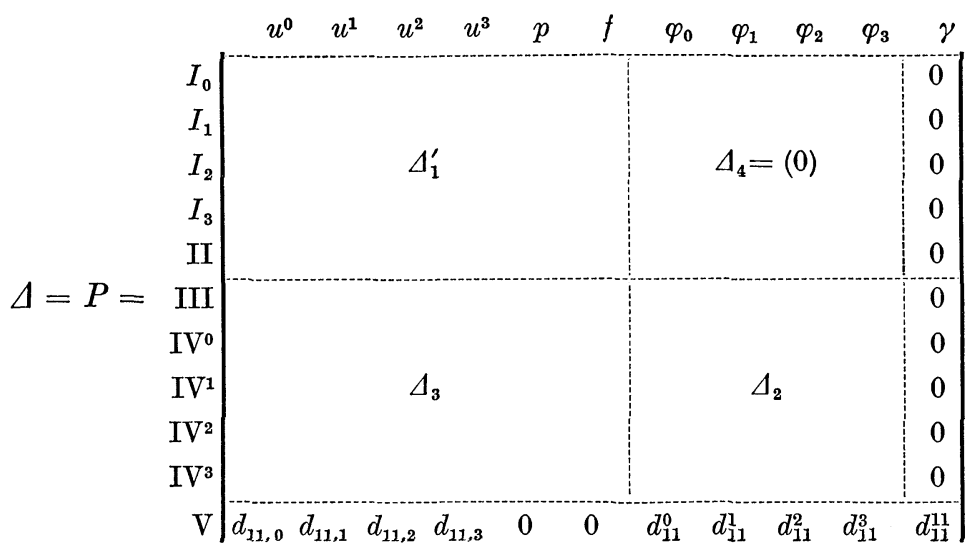

On désigne par $d_{B}^{A}$ et $d_{\bar{\beta}}^{\bar{\alpha}}$ des éléments de $\Delta$ appartenant respectivement à $\Delta_{1}^{\prime}$ et à $\Delta_{2}$ (cf. leurs valeurs paragraphes 6 et 4$)$.

On pose

$$
a=u^{\alpha} \xi_{\alpha} .
$$

On a (cf. V - (1.14) et (1.13))

$$
\begin{gathered}
d_{11}^{11}=u^{\alpha} \xi_{\alpha}=a \\
d_{11, \alpha}=\gamma \xi_{\alpha}+\sigma d_{11, \alpha}^{\prime}, \quad d_{11}^{\bar{\alpha}}=\sigma d_{11}^{\prime \bar{\alpha}}
\end{gathered}
$$

où $d_{11, \alpha}^{\prime}$ et $d_{11}^{\prime \tilde{\alpha}}$ sont respectivement des polynômes du premier et du $2^{\text {ème }}$ ordre en $\xi$. Les mineurs $D_{A}^{B}$ et $D_{\bar{\alpha}}^{\bar{\beta}}$ des éléments $d_{B}^{A}$ et $d_{\bar{\beta}}^{\bar{\alpha}}$ dans $\Delta$ sont

$$
\begin{aligned}
& D_{\bar{B}}^{A}=d_{11}^{11}\left(D_{\bar{B}}^{A}\right)_{\Delta_{1}} \Delta_{2}=a\left(D_{B}^{A}\right)_{\Delta_{1}^{\prime}} \Delta_{2} \\
& D_{\bar{\alpha}}^{\bar{\beta}}=d_{11}^{11}\left(D_{\bar{\alpha}}^{\bar{\beta}}\right)_{\Delta_{2}} \Delta_{1}^{\prime}=a\left(D_{\bar{\alpha}}^{\bar{\beta}}\right)_{\Delta_{\mathrm{a}}} \Delta_{1}^{\prime},
\end{aligned}
$$

où $\left(D_{A}^{B}\right)_{\Delta_{1}^{\prime}}$ et $\left(D_{\bar{\alpha}}^{\bar{\beta}}\right)_{\Delta_{2}}$ désignent respectivement les mineurs de $d_{B}^{A}$ et $d_{\bar{\beta}}^{\bar{\alpha}}$ dans $\Delta_{1}^{\prime}$ et dans $\Delta_{2}$.

D'autre part

$$
\begin{aligned}
D_{11}^{11} & =\Delta_{1}^{\prime} \Delta_{2} \\
D^{11, A} & =D_{\bar{\alpha}}^{11}=0 .
\end{aligned}
$$

Pour calculer les mineurs de $\Delta_{1}^{\prime}$ et $\Delta_{2}$ nous nous placerons dans des axes particuliers et utiliserons les propriétés tensorielles de ces mineurs pour les écrire dans des axes quelconques.

Mineurs de $\Delta_{1}^{\prime}$. Les mineurs des éléments $d_{\alpha}^{\beta}(\alpha, \beta=0,1,2,3)$ de $\Delta_{1}^{\prime}$ forment un tenseur mixte $\left(D_{\beta}^{\alpha}\right)_{\Delta^{\prime} \text {. }}$.

Les mineurs de $d_{A}^{\alpha}$ et $d_{\alpha}^{A}(\alpha=0,1,2,3 ; A=4,5)$ sont respectivement un vecteur covariant $\left(D_{\alpha}^{A}\right)_{\Delta_{1}^{\prime}}$ et un vecteur contravariant $\left(D_{A}^{\alpha}\right)_{\Delta_{1}^{\prime}}$.

Les mineurs $\left(D_{B}^{A}\right)_{\Delta_{1}^{\prime}}(A, B=4,5)$ sont des scalaires. 
Prenons un repère tel que

$$
\begin{aligned}
& \xi_{0}^{\prime}=1 ; \quad \xi_{i}=0, \quad i=1,2,3 \\
& P_{1}=1 ; \quad P_{\alpha}=0, \quad \alpha=0,2,3
\end{aligned}
$$

c'est-à-dire un corepère où $\theta^{0}$ et $\theta^{1}$ étant fixés, $\theta^{2}$ et $\theta^{3}$ sont quelconques. Il suffira donc de calculer dans ces axes $D_{A}^{3}(A=0,1, \ldots 5)$ pour trouver l'expression tensorielle $D_{A}^{\alpha}$.

On trouve pour $D_{A}^{B}(A, B=4,5)$ :

$$
\begin{aligned}
& D_{5}^{5}=(a)^{3}\left\{a r f r_{p}^{\prime}\left(a+\lambda \gamma^{\alpha \beta} P_{\beta} \xi_{\alpha}\right)+r \gamma^{\alpha \beta} \xi_{\alpha} \xi_{\beta}\right\} \\
& D_{4}^{4}=(a)^{4} r^{2} f\left(a+\lambda \gamma^{\alpha \beta} P_{\beta} \xi_{\alpha}\right) \\
& D_{4}^{5}=(a)^{5} \\
& D_{5}^{4}=(a)^{4} r f r_{f}^{\prime}\left(a+\lambda \gamma^{\alpha \beta} P_{\beta} \xi_{\alpha}\right) .
\end{aligned}
$$

Puis pour $D_{\alpha}^{\beta}(\alpha, \beta=0,1,2,3)$ et $D_{A}^{\beta}, D_{\beta}^{A}(A=4,5)$

$$
D_{\alpha}^{\beta}=\xi_{\alpha} D^{\prime \beta}+P_{\alpha} D^{\prime \prime \beta}+\delta_{\alpha}^{\beta} D^{\prime \prime \prime}
$$

où

et

$$
\begin{aligned}
D^{\prime \beta} & =-r^{2}(a)^{3} \gamma^{\lambda \beta} \xi_{\lambda}, \quad D^{\prime \prime \beta}=-r^{2}(a)^{4} f r_{p}^{\prime} \lambda \gamma^{\lambda \beta} \xi_{\lambda} \\
D_{4}^{\beta} & =(a)^{4} r^{2} f \gamma^{\alpha \beta} \xi_{\alpha}
\end{aligned}
$$

$$
\begin{aligned}
& D_{5}^{\beta}=-(a)^{4} r f r_{f}^{\prime} \gamma^{\alpha \beta} \xi_{\alpha} \\
& D_{\alpha}^{4}=(a)^{3}\left\{-r^{2} \xi_{\alpha}\left(a+\lambda \gamma^{\mu \beta} P_{\beta} \xi_{\mu}\right)+a r f r_{f}^{\prime} \lambda P_{\alpha}\right\} \\
& D_{\alpha}^{5}=(a)^{4}\left\{-r a \xi_{\alpha}-r f r_{p}^{\prime} \lambda P_{\alpha}\right\} .
\end{aligned}
$$

On voit sur les expressions obtenues que tous les mineurs $\left(D_{B}^{A}\right)_{\Delta^{\prime}{ }_{1}}$, $A, B=0, \ldots 5$ contiennent en facteur $(a)^{3}$.

Remarque. On déduit de la mise en facteur précédente que les équations des fluides chargés inductifs, où l'on considère le champ électromagnétique et la charge comme connus forment un système hyperbolique strict puisque $\Delta_{1}^{\prime}$ est le déterminant caractéristique de ce système et que

$$
\frac{\Delta_{1}^{\prime}}{(a)^{3}}=a C_{1}^{\prime}(\xi)
$$

est un polynôme hyperbolique strict.

Calcul des mineurs de $\Delta_{2}$. On prend maintenant des axes tels que $u_{0}=1, u_{i}=0(i=1,2,3)$ et $\xi_{1}=1, \xi_{\alpha}=0 \quad(\alpha=0,2,3)$. On trouve ainsi, après être revenu en repère quelconque, en posant $g=g^{\alpha \beta} \xi_{\alpha} \xi_{\beta}$

$$
\begin{aligned}
\left(D_{\bar{\alpha}}^{\bar{\beta}}\right)_{A_{2}}= & \left(g-\lambda a^{2}\right)\left\{\varepsilon \mu g^{2} \delta_{\bar{\alpha}}^{\bar{\beta}}+\lambda g\left(u^{\beta} g-a g^{\beta \mu} \xi_{\mu}\right) u_{\alpha}+\right. \\
& +\lambda a\left(g u^{\beta}-a g^{\beta \mu} \xi_{\mu}\right) \xi_{\alpha} .
\end{aligned}
$$

Tous ces mineurs contiennent en facteur $g-\lambda a^{2}$. 
Remarque. Les équations de l'électromagnétisme dans des fluides en mouvement donné, que la charge soit donnée ou non, ne sont pas hyperboliques strictes puisque le facteur $g$, qui est au carré dans $\Delta_{2}^{\prime}$, n'apparaît pas dans les mineurs de $\Delta_{2}^{\prime}$.

Il résulte des résultats précédents, que, dans le déterminant $\Delta$, les mineurs $D_{B}^{A}$ et $D_{\bar{\beta}}^{\bar{\alpha}}$ contiennent tous en facteur $(a)^{4}\left(g-\lambda a^{2}\right)$.

Autres mineurs de $\Delta$. Les mineurs restant à calculer sont ceux de $\Delta_{3}$, $D^{A \bar{\beta}}$, ceux de $\Delta_{4}, D_{\alpha \bar{\beta}}$ et les mineurs de la dernière colonne de $\Delta, D_{11, A}$ et $D_{11}^{\bar{\alpha}}$.

On voit immédiatement que les mineurs relatifs aux éléments de $\Delta_{3}$ sont nuls (puisque $\Delta=d_{11}^{11} \Delta_{1}^{\prime} \Delta_{2}$ quelque soit $\Delta_{3}$ )

$$
D^{A \bar{\beta}}=0 \text {. }
$$

Les mineurs des éléments de $\Delta_{4}$ sont le produit par $d_{11}^{11}=r a$ de combinaisons linéaires de produits d'un mineur de $\Delta_{1}^{\prime}$ par un mineur de $\Delta_{2}$, dont les coefficients sont les éléments de $\Delta_{3}$ : on voit en effet, par des considérations simples (liées à $\Delta_{4} \equiv 0$ ) que tout terme d'un tel mineur $D_{\alpha \bar{\beta}}$ contient nécessairement un élément de $\Delta_{3}$ et un seul, et que le cofacteur (mineur) d'un tel élément dans $D_{\alpha \bar{\beta}}$ est produit d'un mineur de $\Delta_{1}^{\prime}$ par un mineur de $\Delta_{2}$ et $d_{11}^{11}=r a$.

Développons un mineur $D_{11, A}(A=0, \ldots 5)$ par rapport à sa dernière ligne; les cofacteurs (mineurs) des éléments de cette ligne dans $D_{11, A}$ sont encore des produits d'un mineur de $\Delta_{1}^{\prime}$ par un mineur de $\Delta_{2}$ et un élément de $\Delta_{3}$ pour les éléments $d_{11}^{\bar{\alpha}}$, tandis que pour $d_{11, A}$ ce sont

Enfin:

$$
\left(D^{11, B}\right)_{D_{11,4}}=\left(D_{A}^{B}\right)_{\Delta_{1}^{\prime}} \Delta_{2} .
$$

$$
D_{11}^{\bar{\alpha}}=\Delta_{1}^{\prime} d_{11}^{\bar{\alpha}}\left(D_{\bar{\beta}}^{\bar{\alpha}}\right)_{\Delta_{2}} .
$$

Il résulte de toute cette étude que les mineurs de tous les éléments de $\Delta$ contiennent en facteur $(a)^{3}\left(g-\lambda a^{2}\right)$. Tous contiennent $(a)^{4}\left(g-\lambda a^{2}\right)$ sauf $D_{11, A}$.

Cas inductif $\varepsilon \mu>1$. Il résulte du paragraphe précédent que tous les mineurs de $\Delta$ contiennent en facteur $a^{3}\left(g-\lambda a^{2}\right)$. Le polynôme caractéristique simplifié $\hat{P}(\xi)$ est donc produit de deux polynômes hyperboliques

$$
u^{\alpha} \xi_{\alpha} C_{1}^{\prime}(\xi) C_{2}(\xi) \text { et } u^{\alpha} \xi_{\alpha} C_{2}(\xi) C_{3}(\xi) .
$$

L'existence et l'unicité sont donc assurées dans une classe de Gevrey d'indice $\alpha=2$.

\section{Cas non inductif $\varepsilon \mu=1$}

On voit aisément que pour $\varepsilon \mu=1 \quad(\lambda=0)$ le déterminant caractéristique devient:

$$
\Delta=(a)^{5} \bar{C}_{1}(\xi)(g)^{4}
$$


où $\bar{C}_{1}(\xi)=0$ est le cône classique de l'hydrodynamique relativiste. On vérifie d'autre part sans peine que tous les mineurs de $\Delta_{2}$ contiennent en facteur $(g)^{3}$ donc tous les mineurs de $\Delta$ contiennent en facteur $(a)^{4}(g)^{3}$ sauf $D_{11, A}$ qui s'écrit (les éléments de $\Delta_{3}$ étant nuls)

$$
D_{11, A}=d_{11, \alpha}\left(D_{A}^{\alpha}\right)_{\Delta_{1}^{\prime}} \Delta_{2}, \quad d_{11, \alpha}=\gamma \xi_{\alpha}+\sigma H_{\alpha}^{\lambda} \xi_{\lambda}
$$

et ne contient en facteur que $(a)^{3}(g)^{3}$ si la conductivité $\sigma \neq 0$.

Le résultat sur le système général n'est donc pas amélioré, on a toujours hyperbolicité non stricte avec $\alpha=2$.

Cas de la conductivité nulle. Si $\sigma=0$ (avec toujours $\lambda=0$ ), on vérifie que, compte tenu de $(11.2)$ et $(10.1), D_{11, A}$ contient aussi en facteur $(a)^{4} g^{3}$, le système est hyperbolique strict.

Remarque. Nous avons démontré ce dernier résultat sans l'hypothèse faite dans les travaux antérieurs de la constance dans le fluide du rapport $\gamma / r^{11}$.

\section{Conditions initiales - Vérification des équations tensorielles}

Les équations données par la physique étaient en fait (1.12), (1.14), (1.15) et (1.16). Elles imposent aux données de Cauchy de vérifier les conditions initiales (on prend $x^{0}=0$ pour équation locale de $S$ )

$$
\begin{array}{cccc}
\nabla_{\alpha} G^{\alpha 0}=J^{0} & \text { pour } & x^{0}=0 \\
u^{\beta} u_{\beta}=1 & \text { pour } & x^{0}=0 .
\end{array}
$$

Supposons d'autre part que ces données de Cauchy vérifient la condition de normalisation initiale

$$
\delta \varphi=-\nabla_{\alpha} \varphi^{\alpha}=0 \text { pour } x^{0}=0 .
$$

Montrons que, pour des données de Cauchy vérifiant (12.1), (12.2) et (12.3) la solution trouvée du système $(\Sigma)$ vérifie les équations tensorielles de départ.

En effet: toute solution de I vérifie une équation de la forme ( $\Phi$ est un polynôme des $u^{K}$ et de leurs dérivées premières)

$$
E^{\beta} u_{\beta}=u^{\alpha} \partial_{\alpha}\left(u^{\beta} u_{\beta}\right)+\left(1-u^{\beta} u_{\beta}\right) \Phi=0 .
$$

La condition initiale (12.2) entraîne donc, pour la solution considérée

$$
u^{\beta} u_{\beta}=1
$$

D'autre part, d'après l'identité

$$
\nabla_{\beta}\left(\nabla_{\alpha} G^{\alpha \beta}\right)=0
$$

toute solution des équations IV et $\mathrm{V}$ du système $(\Sigma)$ vérifie

$$
g^{\beta} \nabla_{\beta}\left(\partial_{\varrho} \delta \varphi\right)=0
$$

11 Nous n'avons pas introduit l'entropie $S$ mais les mouvements correspondants au cas étudié ici $\left(\varepsilon \mu=1, \sigma=0, q^{\alpha}=0\right)$ sont nécessairement adiabatiques $\left(u^{\alpha} \partial_{\alpha} S=0\right.$ ) d'après II et III (cf. [5]). 
qui est une équation hyperbolique stricte, du $2^{\text {ème }}$ ordre, linéaire, pour $\delta \varphi$. On déduit de la condition initiale (12.1)

$$
\partial_{0} \delta \varphi=0 \text { pour } x^{0}=0
$$

donc, de (12.1), (12.2), (12.4) pour la solution considérée

$$
\delta \varphi=0 .
$$

On a donc montré que la solution trouvée de $(\Sigma)$ vérifie effectivement les équations tensorielles données.

\section{Cas de la conductivité électrique infinie (conductibilité thermique nulle)}

\section{Equations}

Nous nous placerons encore dans une métrique d'espace temps donnée, pour simplifier l'exposé: le tenseur de Maxwell étant ici symétrique il n'y aurait aucune difficulté à coupler les équations d'Einstein avec les équations des fluides chargés. Ces équations sont (cf. [3], [14])

$$
\begin{aligned}
\nabla_{\alpha} T^{\alpha \beta}=0 & & \text { (mouvement det continuité) } \\
\nabla_{\alpha}\left(r u^{\alpha}\right)=0 & & \text { (Conservation de la matière) } \\
\nabla_{\alpha}\left(h^{\alpha} u^{\beta}-u^{\beta} h^{\alpha}\right)=0 & & \text { (Maxwell) }
\end{aligned}
$$

où

$$
T^{\alpha \beta}=r f u^{\alpha} u^{\beta}-p g^{\alpha \beta}+\tau^{\alpha \beta}
$$

où le tenseur de Maxwell $\tau^{\alpha \beta}$ s'exprime en fonction du champ magnétique $h^{\alpha}$ par

$$
\tau_{\alpha \beta}=\mu|h|^{2} u_{\alpha} u_{\beta}-\frac{1}{2}|h|^{2} g_{\alpha \beta}-h_{\alpha} h_{\beta}, \quad|h|^{2}=-h^{\alpha} h_{\alpha} \geqq 0
$$

compte tenu de l'équation thermodynamique (cf. [12], [5], [11])

$$
T d S=r d f-d p .
$$

L'équation de continuité $u_{\beta} \nabla_{\alpha} T^{\alpha \beta}$ s'écrit:

$$
u^{\alpha} \partial_{\alpha} S=0
$$

on trouve, en utilisant l'équation

$$
\nabla_{\alpha} h^{\alpha}=-h^{\alpha} f^{-1} r^{-1}\left(r \partial_{\alpha} f-T \partial_{\alpha} S\right)
$$

déduite de (13.1) et (13.2) par produit contracté (compte tenu de $h^{\alpha} u_{\alpha}=0$ et $u^{\alpha} u_{\alpha}=1$ ) que le système à résoudre est équivalent à :

II

$$
\left(r f+\mu|h|^{2}\right) u^{\alpha} \nabla_{\alpha} u^{\beta}-\mu h^{\alpha} \nabla_{\alpha} h^{\beta}-2 \mu\left(u^{\alpha} u^{\beta}-\frac{1}{2} g^{\alpha \beta}\right) h_{\lambda} \nabla_{\alpha} h^{\lambda}-
$$

$-\left(\gamma^{\alpha \beta} r+\mu|h|^{2} r_{f}^{\prime} r^{-1} u^{\alpha} u^{\beta}+\mu h^{\alpha} h^{\beta} f^{-1}\right) \partial_{\alpha} f+T\left(\gamma^{\alpha \beta}-\mu h^{\alpha} h^{\beta} f^{-1} r^{-1}\right) \partial_{\alpha} S=0$ 
(équations du mouvement)

III

$$
\nabla_{\alpha}\left(r u^{\alpha}\right)=0
$$

IV $h^{\alpha} \nabla_{\alpha} u^{\beta}-u^{\alpha} \nabla_{\alpha} h^{\beta}+\left(h^{\beta} u^{\alpha} r_{f}^{\prime} r^{-1}-u^{\beta} h^{\alpha} f^{-1}\right) \partial_{\alpha} f+T u^{\beta} h^{\alpha} f^{-1} r^{-1} \partial_{\alpha} S=0$

(équations de Maxwell compte tenu de (11.4)).

Ce système I, II, III, IV de 10 équations aux 10 inconnues $h^{\alpha}, u^{\alpha}, f, S$ est un système quasi linéaire du premier ordre: on pose $m_{k}=1, n_{k}=0$.

\section{Polynôme caractéristique}

Le polynôme caractéristique $P$ est le déterminant $\Delta$ suivant:

où on a posé

$$
P=\Delta=\left|\begin{array}{cccccccccc}
b & 0 & 0 & 0 & -a & 0 & 0 & 0 & c^{0} & d^{0} \\
0 & b & 0 & 0 & 0 & -a & 0 & 0 & c^{1} & d^{1} \\
0 & 0 & b & 0 & 0 & 0 & -a & 0 & c^{2} & d^{2} \\
0 & 0 & 0 & b & 0 & 0 & 0 & -a & c^{3} & d^{3} \\
\lambda a & 0 & 0 & 0 & & & & & \bar{c}^{0} & \bar{d}^{0} \\
0 & \lambda a & 0 & 0 & & \bar{A} & & & \bar{c}^{1} & \bar{d}^{1} \\
0 & 0 & \lambda a & 0 & & & & & \bar{c}^{2} & \bar{d}^{2} \\
0 & 0 & 0 & \lambda a & & & & & \bar{c}^{3} & \bar{d}^{3} \\
r \xi_{0} & r \xi_{1} & r \xi_{2} & r \xi_{3} & 0 & 0 & 0 & 0 & r_{f}^{\prime} a & 0 \\
0 & 0 & 0 & 0 & 0 & 0 & 0 & 0 & 0 & a
\end{array}\right|
$$

$$
\begin{aligned}
a & =u^{\alpha} \xi_{\alpha}, \quad b=h^{\alpha} \xi_{\alpha} \\
\lambda & =r f+\mu|h|^{2} \\
c^{\alpha} & =a r_{f}^{\prime} r^{-1} h^{\alpha}-b f^{-1} u^{\alpha}, \quad d^{\alpha}=u^{\alpha} b f^{-1} r^{-1} T \\
\bar{c}^{\beta} & =\left(r-\mu|h|^{2} r_{f}^{\prime} r^{-1}\right) a u^{\beta}-\mu f^{-1} b h^{\beta}-r g^{\lambda \beta} \xi_{\lambda} \\
\bar{d}^{\beta} & =T\left(-a u^{\beta}-\mu r^{-1} f^{-1} b h^{\beta}+g^{\lambda \beta} \xi_{\lambda}\right)
\end{aligned}
$$

et $\bar{\Delta}$ est le déterminant d'éléments

$$
d_{\alpha}^{\beta}=-\mu b \delta_{\alpha}^{\beta}-r \mu\left(a u^{\beta}-\frac{1}{2} g^{\gamma \beta} \xi_{\gamma}\right) h_{\alpha} .
$$

Par des combinaisons simples de lignes et de colonnes on ramène le déterminant $\Delta$ au suivant

$$
\Delta=a\left|\begin{array}{ccccccccc}
b & 0 & 0 & 0 & 0 & 0 & 0 & 0 & c^{0} \\
0 & b & 0 & 0 & 0 & 0 & 0 & 0 & c^{1} \\
0 & 0 & b & 0 & 0 & 0 & 0 & 0 & c^{2} \\
0 & 0 & 0 & b & 0 & 0 & 0 & 0 & c^{3} \\
0 & 0 & 0 & 0 & & & & & \bar{c}^{\prime 0} \\
0 & 0 & 0 & 0 & & \bar{\Delta}^{\prime} & & & \bar{c}^{\prime 1} \\
0 & 0 & 0 & 0 & & & & & \bar{c}^{\prime 2} \\
0 & 0 & 0 & 0 & & & & & \bar{c}^{\prime 3} \\
r \xi_{0} & r \xi_{1} & r \xi_{2} & r \xi_{3} & r \xi_{0} & r \xi_{1} & r \xi_{2} & r \xi_{3} & r_{f}^{\prime} a
\end{array}\right|
$$


où $\bar{\Delta}^{\prime}$ est le déterminant de

et

$$
d_{\alpha}^{\prime \beta}=d_{\alpha}^{\beta}+\frac{\lambda a^{2}}{b} \delta_{\alpha}^{\beta},
$$

$$
\bar{c}^{\prime \alpha}=\bar{c}^{\alpha}-\frac{\lambda a}{b} c^{\alpha} .
$$

On trouve en développant:

$\Delta=\lambda r a^{2}\left(\lambda a^{2}-\mu b^{2}\right)^{2}\left\{\left(f r_{f}^{\prime}-r\right) a^{4}+\right.$

$$
\left.+\left(r+\mu h^{2} r_{f}^{\prime} r^{-1}\right) a^{2}-\mu f^{-1} b^{2} g^{\lambda \mu} \xi_{\lambda} \xi_{\mu}\right\} .
$$

Le polynôme caractéristique $P=\Delta$ est donc formé par produit des opérateurs hyperboliques suivants:

1) $u^{\alpha} \xi_{\alpha}$ correspondant aux ondes matérielles (plan)

2) $u^{\alpha} \xi_{\alpha} \pm\left(\mu \lambda^{-1}\right)^{1 / 2} h^{\alpha} \xi_{\alpha}$ correspondant aux ondes d'Alfven (plans)

3) $\left(f r_{f}^{\prime}-r\right)\left(u^{\alpha} \xi_{\alpha}\right)^{4}+\left\{\left(r+\mu|h|^{2} r_{f}^{\prime} r^{-1}\right)\left(u^{\alpha} \xi_{\alpha}\right)^{2}-\mu f^{-1}\left(h^{\alpha} \xi_{\alpha}\right)^{2}\right\} g^{\lambda \mu} \xi_{\lambda} \xi_{\mu}$ (correspondant aux ondes hydrodynamiques, opérateur du $4^{e}$ ordre hyperbolique $\mathrm{si}^{12} f r_{f}^{\prime} r^{-1}>1$ (cf. [3], [11])). Le produit de ces opérateurs n'est pas hyperbolique strict (indépendamment du fait que les ondes d'Alfven apparaissent doubles) les plans des ondes d'Alfven se coupant et étant tangents au cône hydrodynamique. Par contre le produit des opérateurs (1) et (3) est encore hyperbolique. $P$ est donc la partie principale du produit de $p=6$ opérateurs.

On a ici $l_{\mathfrak{p}}=5$ donc (notations du paragraphe 9 )

$$
\sup m_{k}-\inf n_{i}=1<l_{p}
$$

d'où le théorème d'existence et d'unicité dans une classe de Gevrey avec

$$
\alpha=\frac{6}{5} \text {. }
$$

Simplification: on voit aisément que tous les mineurs du déterminant $\Delta$ contiennent en facteur $a\left(\lambda a^{2}-\mu b^{2}\right)$. Le système peut donc se mettre sous forme d'un système diagonal dont la partie principale est celle du produit de 3 opérateurs hyperboliques. L'inégalité est vérifiée encore pour $l_{p-p},\left(l_{p-p},=5\right)$, d'où le théorème d'existence et d'unicité dans la classe de Gevrey obtenue par LICHNERowicz

$$
\alpha=\frac{3}{2} \text {. }
$$

Remarque. Considérons les équations linéarisées du système considéré au voisinage de valeurs constantes des $u^{\alpha}, h^{\alpha}, f$. Ces équations sont des équations homogènes à coefficients constants, pour lesquelles on peut prendre $\alpha=\infty$. Elles forment un système hyperbolique strict.

${ }^{12}$ Sauf dans le cas exceptionnel $\mu|h|^{2} f r_{f}=r\left(r f+\mu|h|^{2}\right.$ ) (cf. [3] p. 361). 


\section{Bibliographie}

[1] Abraham, M.: R.C. Circ. Mat. Palermo 28, 27-35 (1909).

[2] Pham Mau Quan: J. Rat. Mech. An. 5, 473-538 (1956).

[3] Bruhat, Y.: Acta Astronautica 6, 354-365 (1960).

[4] Latremoliere, C.: Diplôme d'Etudes Supérieures. Paris 1960.

[5] Pichon, G.: Ann. Inst. Poincaré 2, 21-85 (1965).

[6] Pham Mau Quan: C.R. Acad. Sci. 261, 3049-3051 (1965).

[7] Bruhat, Y., and Pham Mau Quan: C.R. Acad. Sci. 261, 3987-3990 (1965).

[8] Leray, J., et Y. Ohya: Colloque Analyse Fonctionnelle. Liège 1964.

[9] - Math. Ann. 162, 228-236 (1966).

[10] BRUHAT, Y.: Bull. Soc. Math. 86, 155-175 (1958).

[11] Lichnerowicz, A.: C.R. Acad. Sci. 260, 329-334 (1965); Commun. Math. Phys. 1, 328-373 (1966).

[12] TAUb, A. H.: Phys. Rev. 74, 328-334 (1948).

[13] - Arch. Rat. Mech. An. 3, 312-324 (1959).

[14] Lichnerowicz, A.: C.R. Acad. Sci. 260, 4449-4453 (1965); Cours Collège de France 1965/66.

[15] Landau-Lifshitz, L.: Course of theoretical Physics. New York: Pergamon 1960.

[16] Ltchnerowicz, A.: Théories relativistes de la gravitation et de l'électromagnetisme. Paris: Masson 1955.

[17] Coвurn, N.: J. Math. Mech. 10, 361-391 (1961).

[18] Eckart, E.: Phys. Rev. 58, 919-924 (1940).

[19] Bruhat, G.: Cours d'électricité, éd. revue par G. Goddet. Paris: Masson 1961.

[20] Leray, J.: Hyperbolic differential equations. Princeton: Inst. for Adr. Study 1951/52 (Notes miméographiées).

[21] BRuHAT, Y.: J. math. pures appl. (à paraître 1966). 